\title{
A Real Case of Implementation of the Future 5G City
}

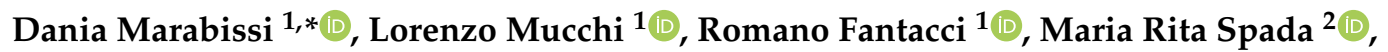 \\ Fabio Massimiani ${ }^{2}$, Andrea Fratini ${ }^{3}$, Giorgio Cau ${ }^{3}$, Jia Yunpeng ${ }^{4}$ and Lucio Fedele ${ }^{4}$ \\ 1 Department of Information Engineering, University of Florence, 50139 Florence, Italy; \\ lorenzo.mucchi@unifi.it (L.M.); romano.fantacci@unifi.it (R.F.) \\ 2 WindTre, 00148 Rome, Italy; mariarita.spada@windtre.it (M.R.S.); fabio.massimiani@windtre.it (F.M.) \\ 3 OpenFiber, 00142 Rome, Italy; andrea.fratini@openfiber.it (A.F.); giorgio.cau@openfiber.it (G.C.) \\ 4 ZTE, 00142 Rome, Italy; jia.yunpeng@zte.com.cn (J.Y.); lucio.fedele@zte.com.cn (L.F.) \\ * Correspondence: dania.marabissi@unifi.it; Tel.: +39-055-2758589
}

Received: 26 November 2018; Accepted: 20 December 2018; Published: 22 December 2018

\begin{abstract}
The fifth generation (5G) of wireless communication systems is considered the key technology to enable a wide range of application scenarios and the effective spreading of the smart city concept. Vertical business use cases, specifically designed for the future 5G city, will have a strong economical and social impact. For this reason, ongoing $5 \mathrm{G}$ field trials have to test newly deployed technologies as well as the capability of $5 \mathrm{G}$ to create a new digital economy. This paper describes the 5G field trial environment that was launched in Italy at the end of 2017. The aim is to evaluate the capability of the $5 \mathrm{G}$ network of supporting innovative services with reference to suitably designed key performance indicators and to evaluate the opportunities offered by these services. Indeed, vertical business use cases, specifically designed for the future 5G city, with a strong economic and social impact, are under implementation and will be evaluated. In particular, the paper provides a detailed description of the deployment of an actual complete integrated 5G network. It shows how 5G is effective enabling technology for a wide range of vertical business and use cases. Indeed, its flexibility allows to satisfy completely different performance requirements of real services. Some preliminary results, obtained during the first phase, are presented for a smart mobility scenario.
\end{abstract}

Keywords: 5G; smart city; field-test; use cases

\section{Introduction}

The evolution of technologies to capture, deliver, and elaborate data with the goal of monitoring, analyzing, and managing the environment is changing our way of living and empowering a wide range of applications and business models that span among different contexts such as smart systems, Industry 4.0, e-Health, intelligent transport systems, and energy management. In the near future, the quality of life of billions of people will depend on the capability of cities of to save energy, reduce harmful emissions, provide smart mobility, improve health services and the living conditions, and increase safety. This poses various challenges that can be overcome in the context of smart systems by adopting disruptive information and communications technologies. In particular, new communication systems should be able to provide ubiquitous access to information and broadband as well as critical services, along with a large variety of scenarios, to a massive number of heterogeneous devices, supporting a huge amount of data: everything will be connected following the Internet of Thing (IoT) paradigm [1]. This is paving the way for a novel and disruptive fifth generation $(5 \mathrm{G})$ communication network, whose challenge is to overcome the fundamental limits of the existing cellular networks, with the aim of guaranteeing a thousand-fold communication capacity increase, extremely low latency, and a massive number of connections in a cost and energy-efficient 
manner [2,3]. 5G systems will be challenged to simultaneously scale up and improve the efficiency of current mobile networks, but also accommodate a wide range of use cases with different and, in some cases, contradictory requirements in terms of coverage, latency, resilience, bandwidth, reliability, mobility, and energy consumption [3]. Indeed, the key difference between 5G and previous generations is the adoption and in-depth integration of a network of verticals, thus enabling the development of new digital services and models. As a consequence, future $5 \mathrm{G}$ networks are faced with the challenge of supporting three main categories of use cases [4]: (i) enhanced mobile broadband (eMBB), which delivers gigabytes of bandwidth on demand, (ii) massive machine-type communication (mMTC), which connects billions of machines, and (iii) ultra reliable and low-latency (URLL) communication, which allows immediate feedback with high reliability for critical services. In order to satisfy all these diverse use cases and their demands, 5G networks call for such qualities as adaptation, scalability, re-reconfigurability, virtualization, and self-organization.

The Third Generation Partnership Project (3GPP) started the 5G standardization process with initial study items as part of Release 14. The standardization was divided in two phases: (i) Phase 1-Release 15-provides the set of features, functionality, and services needed for deploying a commercially operational system, (ii) Phase 2-Release 16-will incorporate more functions to extend the $5 \mathrm{G}$ capabilities progressively, to support more services, scenarios, and higher frequency bands (to be completed in 2019). The aim of this division in two phases is to complete initial specifications on time and allow for commercial deployment and service launch in 2020 [3]. Together with standardization, field tests under real-world conditions are a significant step toward the launch of $5 \mathrm{G}$ networks. Indeed, these allow one to determine how to best leverage the new capabilities of users and to evaluate the key performance indicators (KPIs). In particular, KPIs represent the performance requirements that must be met in each application scenario, such as cell and user peak data rate, data and control plane latency, mobility capabilities, reliability, availability, and density of devices [4].

\subsection{Related Works}

Field tests and trials are of paramount importance to measure and verify the actual achievements of the new 5G systems. Currently, there are several ongoing field trials. The large part of these has as goal-that of proving the effectiveness of 5G New Radio (NR) key technologies such as 3D beamforming, massive MIMO (Multiple Input Multiple Output), new air interfaces, multiple frequency bands, and advanced core solutions [3,5-7]. The majority of the field-trials focus on eMBB scenarios over a wide range of frequencies, and key observations are related to the downlink capacity and the radio propagation at high frequencies. Nevertheless, there is limited information in the literature about trials of mMTC and URLLC scenarios and of complete integrated systems and networks. Moreover, as stated before, 5G evolution will not be limited to mobile broadband, but will bring new capabilities both in terms of network and services, thus opening the way to new business models where operators and players will define new collaboration strategies. Toward this goal, it is needed to verify how $5 \mathrm{G}$ networks are able to efficiently support a wide variety of use cases. Several papers in the literature present high level 5G frameworks able to support, for example, smart city $[8,9]$, smart energy [10], and public safety [11-13] applications. Moreover, requirements and challenges of 5G systems to support specific use cases are presented in different papers as in [14], in [15] for industrial automation, in [16] for road users' connectivity, and in [10] for smart grids. Use cases that are under test in the Finnish 5G field trial are presented in [17], without a detailed description of the actual $5 \mathrm{G}$ implementation.

There are few demonstrations whose goal is to show the potential of a 5G system to support new applications and services, while there are several analytic and simulation studies. Moreover, in general, these demonstrations focus on a single very specific use case. For example, in [18], the ability of 5G of supporting virtual reality is under investigation. Measurements on specific performance requirements for connected mobility (i.e., latency, handover time and coverage) have been provided in [19] for a 
commercial 4G network. The paper shows that this technology is not suitable for such use cases and indicates $5 \mathrm{G}$ as the best future candidate for its potentialities.

\subsection{Our Contribution}

This paper presents the testing and field trial environment for 5G that was launched in Italy at the end of 2017, in the cities of Prato and L'Aquila. Differently from other papers that present other field tests, this paper does not focus on a single key feature, on a specific type of scenario, or on an isolated network portion. The presented field trial environment is one of the first experiences of a complete integrated network implementing the most important $5 \mathrm{G}$ disruptive technologies. This paper presents a detailed description of the effective implementations, considered technologies, and incremental features that will be added during the project and 3GPP standard evolution. Moreover, as 5G technology is not only an evolution of current mobile networks but also a platform for a wide range of smart services and applications, this paper shows how completely different use cases, with completely different KPIs, can be accommodated on the same platform thanks to $5 \mathrm{G}$ flexibility and reconfigurability. Indeed, the aim of the field trial environment is to test newly deployed technologies as well as the capability of $5 \mathrm{G}$ to create a new digital economy with a ubiquitous network that will connect people, things, and services. Vertical business use cases, specifically designed for the future 5G city that can have a strong economical and social impact, will be tested. In particular, the aim is to evaluate the capability of the 5G network of supporting innovative services with reference to suitably designed KPIs, and to evaluate the opportunities offered by these services. The tests are conducted using the experimental licenses on the 3.6-3.8 GHz frequency band released by the Economical Development Minister (MISE-Ministero per lo Sviluppo Economico) after a public competition responding to the European Commission's request for a timely and efficient 5G deployment as a strategic opportunity for Europe.

The entire experimental project will last 2.5 years (up to June 2020) with a pre-commercial experimentation during the last year. In particular, according to the 3GPP standardization process, the final 5G architecture will be tested starting from 2019 when almost all 5G features will be defined.

The field trial is led by WindTre and OpenFiber, who joined their knowledge, infrastructures, and economical efforts. Both WindTre and OpenFiber are leading companies in the communication sector and own relevant infrastructures of radio networks and optical fiber backbones. Moreover, several other companies, utilities, research centers, and public administrations are involved as partners. Among these, ZTE provides NR access, core networks, and transport technologies.

\subsection{Field Trial Details}

As stated before the 3GPP completed (at the end of 2017), the first version of the 5G standard ready for deployment that requires fallback to Long Term Evolution (LTE) networks for partial operation. This 5G solution is called non-standalone 5G NR (NSA 5G NR). Release 15 (June 2018) has defined the first draft of the Stand Alone (SA) 5G network architecture.

The project 5G has been organized in phases in order to follow the standardization process:

\section{Set-up phase}

- Enhanced studies -5G new technologies are studied and evaluated, in order to define possible algorithms and strategies to be implemented in the successive field tests;

- Lab tests-5G new technologies and devices are tested in the ZTE Research and Innovation laboratories, with particular reference to 5G NR.

\section{Roll-out phase}

- NSA tests-all network elements and basic network functions are tested on the field with the deployment of several NR base stations (BSs), while the core network $(\mathrm{CN})$ is based on existing LTE-CN following the NSA network architecture. 
- $\quad S A$ tests-definition and deployment of SA network architecture on the field with the deployment of the $5 \mathrm{G}-\mathrm{CN}$.

\section{Service phase}

- Service tests - test of innovative smart systems and services provided on the 5G network infrastructure and validation of KPIs.

The project timeline related to 3GPP standardization process is represented in Figure 1. It includes the currently existing assets (green) as well as upcoming assets (gray).

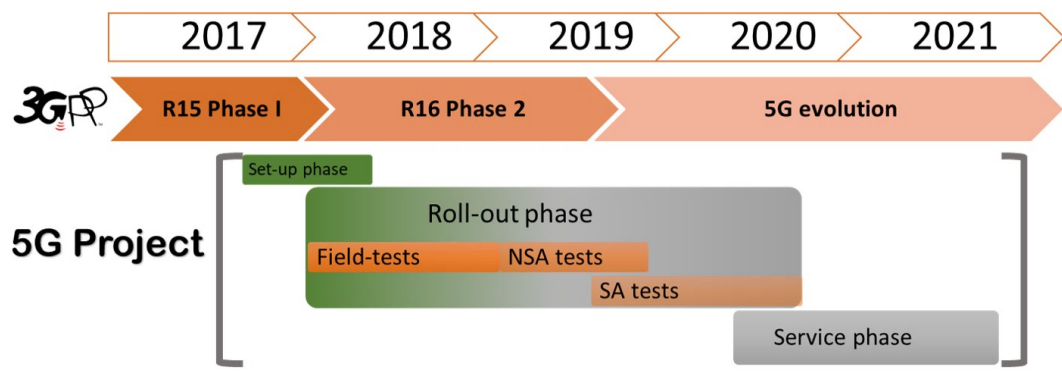

Figure 1. Project timeline.

In particular, while current field deployments refer to the latest defined standard version (Release 15), in the successive phase, new features of Release 16 will be included during the next year. Moreover, some enhanced studies are carried out on advanced features of Releases 15 and 16, even if these will not be deployed during the field trials.

The paper is organized as follows. The 5G network infrastructure is presented in Section 2 with reference to the different deployment phases providing details on implemented technologies. Successively, the use cases of the service-tests phase are briefly described with relative service KPIs in Section 3. More details are provided for the smart mobility use case.

\section{5G Network Architecture}

One of the key drivers of $5 \mathrm{G}$ systems is the need to support a wide variety of vertical markets that originate extremely different use cases and, consequently, extremely different requirements. Such a wide range of requirements cannot be met through a common network setting, and deploying individual mobile network solutions for each use case is unfeasible. Hence, there is a general consensus in considering network slicing as a key element of 5G networks. With network slicing it is possible to compose and manage dedicated end-to-end logical networks with specific functions, without losing the economies of scale of a common physical infrastructure [20]. This is the approach considered in the $5 \mathrm{G}$ experimental test-bed, described here.

The network is logically divided in three layers as represented in Figure 2: (i) physical infrastructure, (ii) network function, and (iii) service layers. The physical infrastructure layer is in charge of the control and the management of the physical resources (i.e., radio access networks (RANs), computing, storage, $\mathrm{CN}$ ), while the second layer configures the network functions (both in the user and control plane) customized for each slice. The third layer translates the services in the slice's configurations in terms of quality of service (QoS) requirements, service level agreements (SLAs), and needed functionality. The three layers are managed by an orchestrator that maps the resources available at different layers to the slices.

In particular, two different network models will be implemented:

- the "open access" model proposed and deployed by Open Fiber in the city of Prato, where the operator that deploys the network is only an infrastructure provider (InP) that offers network 
slices to virtual operators, enterprises, and public administrations with the goal of providing services to the end user;

- the "vertically integrated operator" model proposed and deployed by Wind-Tre in the city of L'Aquila, where the InP is also the operator that provides services to the end users (no third parties are involved). This means that different network slices are owned and managed by the InP itself.

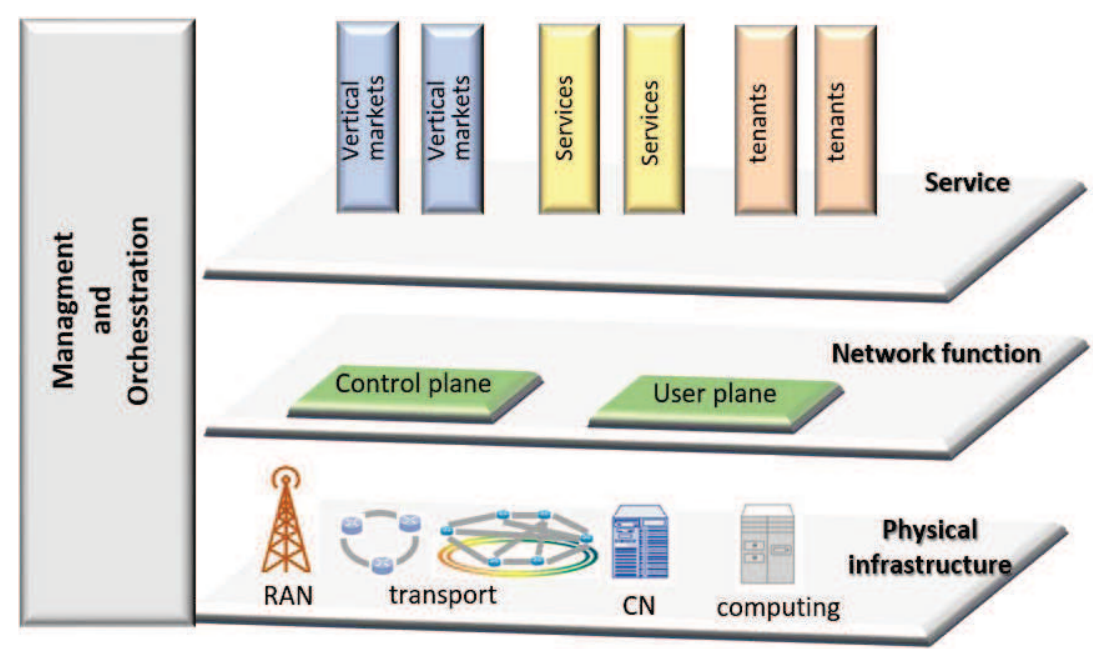

Figure 2. Network slicing conceptual architecture.

\subsection{G Physical Infrastructure}

\subsubsection{Set-Up Phase}

During the set-up phase, enhanced-studies are devoted to investigating possible algorithms and strategies to efficiently and effectively implement new technologies and paradigms that are considered key enablers of 5G network capabilities. Simultaneously, lab tests are carried out to test basic RAN functions. RAN is deployed following the Cloud-RAN approach, which is based on the separation of the radio frequency (RF) elements of the BS (called the active antenna unit (AAU)) from the processing of the baseband signal (called the base band unit, (BBU)), which is centralized in a single point or virtualized in the cloud. AAU can be deployed on multimode networks while working on multiple frequencies. A cloud RAN allows easier operations using a simpler RF equipment at the network edge, while the RAN intelligence is centralized in the BBU. AAUs and BBUs must be connected with high-speed and low-latency links called fronthaul. Toward this goal, the decision is to adopt the Common Public Radio Interface (CPRI) specifications [21] for transmitting digital radio over fiber. CPRI is a serial line interface transmitting constant bit rate data over a dedicated channel. During initial tests the physical architecture is made by a single BBU with a single AAU connected to a channel emulator and to basic user equipment. The 5G NR under investigation is based on an improved air interface that does the following:

- allows operation in multiple frequency bands (3GPP-Release 15). The breakthrough in this context is to allow a device being simultaneously connected to a high frequency layer that carries only user plane traffic and to a lower-frequency layer that provides ubiquitous connectivity to both user and control planes;

- uses massive MIMO techniques (3GPP-Release 15). The BBU and AAUs enable computationally intensive operations to support an unprecedented number of antenna elements. Moreover, efficient beamforming schemes are considered to fully exploit massive MIMO capabilities;

- uses non-orthogonal multiple access (NOMA) (3GPP-Release 16). The overhead due to orthogonal transmissions becomes very large when the number of connected devices increases and can 
introduce unacceptable latency. For this reason, NOMA approach is considered for massive machines access;

- relies on a dense network deployment (3GPP-Release 15), a multi-tier heterogeneous architecture where high-power macrocells are overlaid by a large number of low power small cells that provide high data rates and dedicated capacities to homes, enterprises, and urban hot-spots and to offload excess traffic from the macrocell.

\subsubsection{Roll-Out Phase-NSA Architecture}

During the first period, the roll-out phase focuses on NSA architecture: several BBUs with one or more AAUs are deployed on specific areas of the two cities and connected to the LTE-CN in order to evaluate the transport network, including fronthaul and backhaul links. Transport network is managed via software, thus allowing a flexible reconfiguration of all the network elements using the FlexE [22] standard that allows for flexible use of the links capacity, making the physical layer transparent to the service layer. Connections of BBUs is provided using 100G Ethernet rings, while connections with the core network is supported by using optical links.

The NSA 5G NR utilizes the existing LTE radio and CN as an anchor for mobility management and coverage while adding a new 5G carrier. More specifically, in this case, the 5G NR is connected to the LTE-CN by means of the LTE-RAN. This configuration reflects the 3GPP NSA deployment scenario Option 3x [23]. In this configuration, even if the 5G coverage is initially jeopardized, services can be provided thanks to the $4 \mathrm{G}$ coverage. Hence, there is a strong interest in evaluating the coexistence and the integration with the already existing $4 \mathrm{G}$ network and the portability of the services that currently can be provided by means the $4 \mathrm{G}$ network. Moreover, dual-connectivity tests are ongoing.

Although Network slicing and SDNs are not officially part of the tests planned by the Ministry of Economic Development, the NSA phase represents a precious chance to test network slicing and a software-defined network (SDN) [24] technology that allows one to control and coordinate different network slices on a common physical infrastructure.

During the NSA phase basic network functions are validated and related KPIs are as follows:

- control plane latency: time to switch from an IDLE or INACTIVE state (low battery consumption) to an ACTIVE state (data transfer). Expected value: $\leq 20 \mathrm{~ms}$ for URLL and eMBB scenario. Reference International Telecommunication Union (ITU) 5G requirement: $\leq 20 \mathrm{~ms}$.

- user plane latency: time to successfully transmit an application-layer packet/message, from the input level of the protocol 2/3 to the output level of the protocol 2/3, for both the uplink (UL) and downlink (DL) radio interface. Expected value: $\leq 4 \mathrm{~ms}$ for eMBB scenarios. Reference ITU-5G requirement: $\leq 1 \mathrm{~ms}$ user plane latency for URLLC and $\leq 4 \mathrm{~ms}$ for eMBB.

- cell peak data rate: maximum theoretical cell data rate. Expected value: $\geq 4 \mathrm{Gbps}$ (DL), $550 \mathrm{Mbps}$ (UL). Reference ITU-5G requirement: $\geq 10 \mathrm{~Gb} / \mathrm{s}$ cell peak data rates for eMBB.

\subsubsection{Roll-Out Phase-SA Architecture}

During the last part of the roll-out phase, the SA 5G NR architecture will be deployed. This implies full user and control plane capability for 5G NR, utilizing the new 5G CN architecture. In particular, Option 2 envisaged by 3GPP [23], will be considered in the network deployment where 5G NR devices are directly connected to the new $5 \mathrm{G} \mathrm{CN}$. This choice implies simple implementation and independence on $4 \mathrm{G}$ network deployments that allow for higher freedom to the 5G operators. At the same time, this solution is challenging because it requires that the $5 \mathrm{G}$ end-to-end network is almost completely defined and developed before the end of the project, and substantial investments are needed to provide suitable territory coverage. In this phase, it is interesting to validate KPIs related to mobility and handover. In particular,

- mobility interruption time: maximum no activity time during handover (the terminal cannot exchange data with the base station). Expected value: $1 \mathrm{~s}$. 


\section{Future 5G City Use Cases}

As stated before, the main objective of the demonstration is to show the potential of a $5 \mathrm{G}$ system to support new applications and services. In particular, the focus is toward new services that can be deployed in a metropolitan environment and can significantly affect our lives.

\subsection{G Use Cases and Services}

$5 \mathrm{G}$ technology is designed to providing a wide range of services differing in requirements and in the type of devices, going beyond human-type communications. While current services are mainly focused on the personal communication paradigm, i.e., voice, instant messaging and social networks, multimedia streaming, and Internet browsing, in the future these will evolve towards the IoT paradigm. As a consequence, new 5G macro-applications will appear:

- industrial automation: integration of sensors, robotics, and remote control.

- transportation: assisted driving, autonomous driving, smart sensors on vehicles, information on traffic and dangers, and visual empowered services (see-trough).

- digitalization of the living environment: smart home, smart city, street-light illuminations, smart energy grids, smart water and gas distribution, waste recycling, environment sustainability, and cultural heritage empowerment.

- public safety services: information system for police, and alert and management systems in case of natural disasters.

- tele-presence services: virtual reality, holograms, and virtual offices.

- other systems of public utility: health-care, education, and civic participation.

The International Telecommunication Unit (ITU) has grouped a wide variety of use cases in three broad categories [25]:

1. Enhanced Mobile BroadBand (eMBB). 5G technology has to provide a high data rate and a low latency to step mobile services up a level, by allowing always-on always-connected mobile terminals with real-time reactivity. Seamless coverage and high mobility are needed in large areas with higher data rates than today, while a high user density and very high traffic capacity are needed in hotspots. Head-mounted displays for immersive experiences (augmented and/or virtual reality) will become a service that can be deployed everywhere.

2. Massive Machine Type Communication (mMTC). This is one of the most expected 5G-enabled services: the possibility to interconnect a high density of devices (hundreds of thousands of devices per square kilometer). Literally everything can radiate and be connected. Typically these devices are characterized by a relatively low volume of data to transmit and by stringent power consumption constraints, and they must be low cost. For example, the Internet of Medical Things (IoMT) will provide new opportunities for both enterprises and users, globally.

3. Ultra Reliable Low Latency Communication (URLLC). In this case, reliability, latency, and availability are the main requirements. The focus is on critical communications whose timely and accurate behavior impacts safety: monitoring and control must occur in real time. This is, for example, the case in industrial process control, automated vehicles, public protection, and disaster relief applications. Industrial communications will benefit enormously by the ultra reliable inter-connection as well as by the very low latency. Control of critical infrastructure or automated vehicles are only two of the top services that 5G will enable. For example, the vehicle-to-everything (V2X) technology will interconnect vehicles, pedestrians, road infrastructure and cloud, opening the possibility to use unmanned vehicles on the road.

To have a differentiated while optimized wide variety of services, 5G must simultaneously support different combinations of stringent KPIs such as a high data rate, very low latency, high reliability and availability, great positioning accuracy, density of connected devices and network capacity. ITU has 
defined the minimum technical performance requirements for 5G [26]. Main KPIs are reported in Table 1 along with the use case for which they are relevant. These KPIs will be monitored according to the specific use case during the service tests phase, as specified later.

Table 1. KPIs to assess the performance of $5 \mathrm{G}$ use cases in the field trials.

\begin{tabular}{cccc}
\hline KPI No. & Description & Value & Key Use Case \\
\hline K1 & Density (devices $\left./ \mathrm{km}^{2}\right)$ & $\geq 10,000$ & mMTC \\
K2 & Mobility $(\mathrm{km} / \mathrm{h})$ & up to 500 & eMBB \\
K3 & Peak data rate & DL:20 Gbps, UL:10 Gbps & eMBB \\
K4 & User Data Rate (Mbps) & DL:100, UL:50 & eMBB \\
K5 & User plane Latency (ms) & $4($ eMBB), 1 (URLL) & eMBB, URLL \\
K6 & Control plane Latency (ms) & 20 & eMBB, URLL \\
K7 & Reliability & frame error rate $<10^{-5}$ & URLL \\
K8 & Availability $(\%)$ & $>99$ & URLL \\
\hline
\end{tabular}

\subsection{The $5 G$ Project Use Cases}

This section provides a brief description of the use cases that will be deployed during the project, while more details and some preliminary results are given for the smart mobility use case in the next section. All use cases have been suitably designed in each city in accordance with all partners and public administration interests. Table 2 reports for each use case (i) the related class of ITU use case, (ii) the KPIs that will be evaluated with reference to the previous table, and (iii) which city will be tested.

Table 2. Use cases in Prato and L'Aquila.

\begin{tabular}{cccc}
\hline Use Case & ITU Classes & KPIs & City \\
\hline e-Health & eMBB, mMTC, URLLC & K4, K5, K6, K7, K8 & Prato, L'Aquila \\
Smart industry & mMTC, URLLC & K1, K5, K6, K7, K8 & Prato \\
Smart grid & mMTC & K1, K8 & Prato \\
IoT and sensors & mMTC & K1, K8 & Prato \\
City surveillance & eMBB & K3, K4, K8 & Prato \\
Virtual reality for cultural heritage & eMBB & K3, K4, K7 & Prato, L'Aquila \\
Structural health monitoring & eMBB, mMTC & K1, K3, K4, K5 & L'Aquila \\
Agriculture 2.0 & eMBB & K2, K4 & L'Aquila \\
Smart safety for smart city & eMBB & K3, K4, K8 & L'Aquila \\
Smart mobility: High connected EV road monitoring & mMTC, URLLC & K1, K2, K5, K6, K7, K8 & Prato \\
Smart mobility: advanced viability & mMTC, URLLC & K1, K2, K5, K6, K7, K8 & L'Aquila \\
\hline
\end{tabular}

\subsection{1. e-Health}

The aim is to develop a platform for telemedicine, telemonitoring, and analysis of behavioral habits, capable of enabling processes to guarantee continuity of care and assistance. The collected data and the possibility of remote assistance allow doctors to establish an interactive relationship with patients, providing them with a personalized therapeutic path. This solution rationalizes costs and promotes the creation of high-added-value services able to meet the needs of both individual and corporate customers, and provides a series of health services that can be used remotely. Drones for the rapid transport of medicines and medical equipment will be also implemented.

\subsubsection{Smart Industry}

This use case is devoted to the development of a digital smart industry platform able to meet the needs of industrial customers. The platform provides a complete portfolio of added value services as envisaged by the directive Industry 4.0 for the optimization of production processes, energy efficiency, maintenance, and operation. In particular, the experimentation proposes to realize an integrated IoT solution that allows one to manage data from various sensors/actuators and processes in the industrial sector. This allows one to create integrated Industry 4.0 systems that work in a manufacturing as a service (MaaS) perspective. 


\subsubsection{Smart Grids}

Different sensors (acoustic, accelerometers, temperature, ozone and other gases, deterrents for copper theft) will be installed on a selected electrical grid, in some secondary substations, and on the connected low voltage lines, for monitoring the network and for predictive maintenance and infrastructure security. A management architecture inspired by the blockchain protocol will be built to enable new services and management methods of the load and generation assets, in order to facilitate the integration of distributed generation in medium- and low-voltage networks. The 5G will then provide radio access to the peripheral plants (primary and secondary cabins) for the construction of an integrated network that has a core component and that can also include a mix of different connectivity solutions (fiber, $\mathrm{xDSL}$, etc.).

\subsubsection{IoT and Sensors}

The aim is to utilize smart sensors, and in general the IoT paradigm, for multiple fields of application. In particular, an IoT data management platform will be developed on which to graft control algorithms and represent current and forecast values on dashboards, including notifications and alerts in real time. The 5G network will enable the direct communication of objects (sensors, cameras, etc.), thus speeding up and optimizing the implementation processes. Specific objectives are as follows:

- real-time remote control of the operating conditions of processes or plants;

- remote control of heavy machinery in hazardous environments;

- logistics optimization;

- tracking of products.

One of the most important applications is the collection of environmental data, including those related to the health of the workplace, data for the management of a smart city, and data deriving from remote reading systems of gas meters, water, and electricity.

\subsubsection{Video Surveillance}

One goal is to create a capillary video surveillance system in a municipal territory: every point at which a power supply is available becomes a possible installation point for a camera. The high throughput of 5G technology enables the use of ultra-high-resolution cameras, and their control/management can be performed remotely in real time. The dynamic use of non-sanctioning car-plate visual control will allow for a more widespread and targeted control over the territory. At the same time, together with the use of thermal cameras, the abandonment of hazardous waste can be monitored generating alerts with the exact position of the area.

\subsubsection{Augmented and Virtual Reality for Cultural Heritage}

Another aim is to evaluate the enhancement of cultural heritage, thanks to new experiences provided by immersive 360-degree visits of churches, museums, historic buildings, and archaeological sites, with digital content displayed through virtual reality (VR) and augmented reality (AR) viewers. Smart Glass and live streaming online or social networks can be used to enhance the guided visits of cultural heritage sites. It will be possible to enjoy content by means of VR and AR inside the Pecci Museum and the Tissue Museum in Prato, and inside museums (MuNDA museum), historic buildings, churches, and archaeological sites in L'Aquila.

\subsubsection{Structural Monitoring of Buildings and Infrastructures}

Another objective is the implementation of a monitoring service for public/private buildings and civil infrastructures, which can also be applied to cultural assets. In particular, the most significant structural parameters must be fully and immediately visible, thus reporting any anomalies and critical situations even in emergency conditions (e.g., earthquakes). The experimentation scenarios will be 
two, one based on the use of sensors installed into the buildings and the other one on the use of drones connected to the $5 \mathrm{G}$ network. The use of drones allows the rapid, efficient, and precise collection of very-high-resolution frames that, after being processed through the use of neural networks, can be used to determine the current state of the health of buildings. Data can be compared to previously collected data to determine any damage.

\subsubsection{Agriculture 2.0}

Another aim is the certification and enhancement of the Made in Italy brand, with the application of innovative solutions for traceability of the production chain in the Agro-Food sector. Technology based on blockchain and precision agriculture will be used, e.g., drones connected to the $5 \mathrm{G}$ network for transmission in time real of trusted data that reinforce the authenticity and consistency of information, to ensure the authenticity of the production. The experimentation involves the entire production chain: cultivation, irrigation, fertilization, and harvesting.

\subsubsection{Smart Safety for Smart Cities}

Another objective is to ensure a greater level of safety for citizens by using innovative technologies that allow security forces to immediately determine the emergency status of an event and to simplify/optimize coordination and intervention activities. Security agents will be equipped with smart glasses connected to the $5 \mathrm{G}$ network and remote-controlled drones equipped with cameras for a top view, in order to offer the best support for effective decision making in the management of citizens' safety, in emergency and danger situations as well as in daily operations.

\subsubsection{Security Issues}

Although 5G standards are still being defined and large-scale commercial implementations are not expected before 2020, a sure challenge is that the market acceptance and success of 5G services will require a gradual improvement in security. Any perception that $5 \mathrm{G}$ technology relies on uncertain security aspects will decrease user confidence and will expose system operators and service providers to lower investments and to legal and regulatory implications. In this context, mobile security and managed security services are one of the most intensive sectors in terms of innovation, to respond to the challenges in the field of cyber-security inherent to digital transformation for the Communication Service Providers and their customers. In particular, agility and automation are the basic elements of digital transformation for the Communication Service Provider and its customers. Fundamental to any service, security must integrate into these agile and automated ecosystems.

Furthermore, the $5 \mathrm{G}$ promises to reshape services and promote innovation. With increased bandwidth, multitenant edge computing, and several innovative services, from high-bandwidth connectivity to machine learning and AI, security has never played a major role in the mobile operator's infrastructure. The protection of the virtual infrastructure of mobile operators in $5 \mathrm{G}$ requires a powerful, scalable, and agile security.

In summary, the main $5 \mathrm{G}$ security challenges are as follows:

- $\quad$ protecting a virtual and agile mobile core with 5G;

- integrating security services into new innovative services offered through 5G such as mobile edge computing (MEC) and network slicing;

- providing a complete ecosystem of security services to generate new revenue, create competitive advantage, and increase customer loyalty.

\subsection{Smart Mobility}

Smart mobility is expected to support different social challenges, so having safe, efficient, reliable, and green freight and passengers transport is a widely shared policy perspective. In this context, $5 \mathrm{G}$ is envisaged as the ultimate platform to enable smart mobility solutions, and cellular-vehicle 
to everything (C-V2X) functionality will be included as part of the cellular chipsets embedded into vehicles for their vehicle to network (V2N) communications. Hence, the smart mobility vertical domain is one of the key driving sectors for evolution towards $5 G$ and one of the most important use cases under evaluation in the $5 \mathrm{G}$ project. The smart mobility platform that is under deployment is divided into two different use cases: the "high connected EV road monitoring" and the "advanced viability." The main goals are the following:

1. Highly connected EV road monitoring: An electric car park and charging points will be deployed with the aim of monitoring, during normal daily activities, the state of the road surface (presence of holes, slope, traffic conditions, etc.) through the installation in cars of a blackbox containing a 5G module for real-time data transmission to post-processing systems. A differential GPS, placed in electric cars, will map with centimeter precision the geographic coordinates of the holes detected during the normal use of vehicles.

2. Advanced viability: Connected vehicles will exchange data with other vehicles and with a control center where data traffic information is smartly integrated with weather conditions, road status, and other information provided by IoT sensors in the city. The aim is of improving the safety of vehicles and drivers, comfort, and driving style and reducing road traffic, congestion, and consequent pollution, using real time information.

The reliability of the 5G network will offer advanced real-time communication services also in very high density of network nodes that can occur for example for traffic jam or an emergency situation. While 5G network deployment is ongoing, some preliminary results on the smart mobility scenario have been achieved as an outcome of enhanced studies. The aim is the evaluation of advanced techniques that will not necessarily be implemented in the field tests but that represent a step towards the full achievement of ITU-5G requirements. These preliminary results are presented in the next subsection, referring to different key technologies for the smart mobility scenario.

\section{Key Technologies}

The considered smart mobility platform relies on some key technologies that are currently under study. In this section, some hints and results are presented. Not all the key technologies described in this section are planned to be used in the tests officially listed in the 5G program of the Ministry of Economic Development. Anyway, additional tests can be run by universities or research centers participating to the $5 \mathrm{G}$ program.

Network Slicing. V2X communications under the 5G network will be provided by specific network slices. However, nowadays efficiently managing slices is a challenge in resource allocation. Hence, the ongoing studies focus on practical algorithms that allow one to solve the problem taking into account computational complexity and the ability to reconfigure and migrate slice resources in accordance with the variable vehicular environment. At the network function layer, the aim is to realize an optimal matching among functions and slices in accordance with their descriptors. Differently, at the infrastructure level, the main challenge is how to virtualize and divide the RAN into different slices. Indeed, while at the $\mathrm{CN}$ level the network can be scaled up adding more hardware resources, RAN has to face with spectrum scarcity. Moreover, in the vehicular context, RAN must be flexible enough to dynamically accommodate radio resources to the slices taking into account the coexistence of cellular (V2N) and direct communication, such as vehicle-to-vehicle (V2V) communication. Two solutions are under evaluation. In the first one, the RAN scheduling has two stages: one slice-specific and another cell-specific. Slice-specific scheduling works on virtual resources and is in charge of the scheduling of resources among the users of the slice. Each slice can adopt a different scheduling algorithm. Virtual resources are then mapped into physical resources by the cell-specific scheduler that is in charge of the division of the resources among the slices, in general guaranteeing a minimum capacity to each slice [27]. The second solution is a multiservice scheduling that jointly performs intra- and inter-slices allocations. This has to take into account users' capacity requests, channel conditions, and priority 
(depending on the slice), so it requires the solution of complex multiobjective problems, even if it can provide higher flexibility and efficiency. In order to investigate RAN slicing challenges and solutions, some studies have been carried out by the University of Florence. We show here some achieved results. Figure 3 shows the performance in terms of the outage rate (i.e., the total amount of users' data rate requests not satisfied) obtained by using a multiservice scheduler and two two-stage schedulers that first divide the resources among slices proportionally (i) to the mean number of users and (ii) to the priority, respectively, and then allocate intra slice resources using a proportional fair approach. One slice is devoted to vehicular traffic and has higher priority, and the mean data rate of the users is half of that of the other slice that is devoted to general broadband (BB) data traffic. Results are given as a function of the ratio of the number of users. In detail, the number of vehicular users is a random Poisson-distributed variable with a mean value in the range [25-100], while the mean value of the BB users is fixed at 50 . We can see that using a multiservice solution allows for a better management of resources both in terms of the total outage rate of the system and in terms of isolation. Indeed, the outage rate of vehicular users is not affected by changes in the other slice. As a conclusion, a single multiservice scheduler can provide efficiency with a suitable level of isolation and thus is a good solution to be implemented.

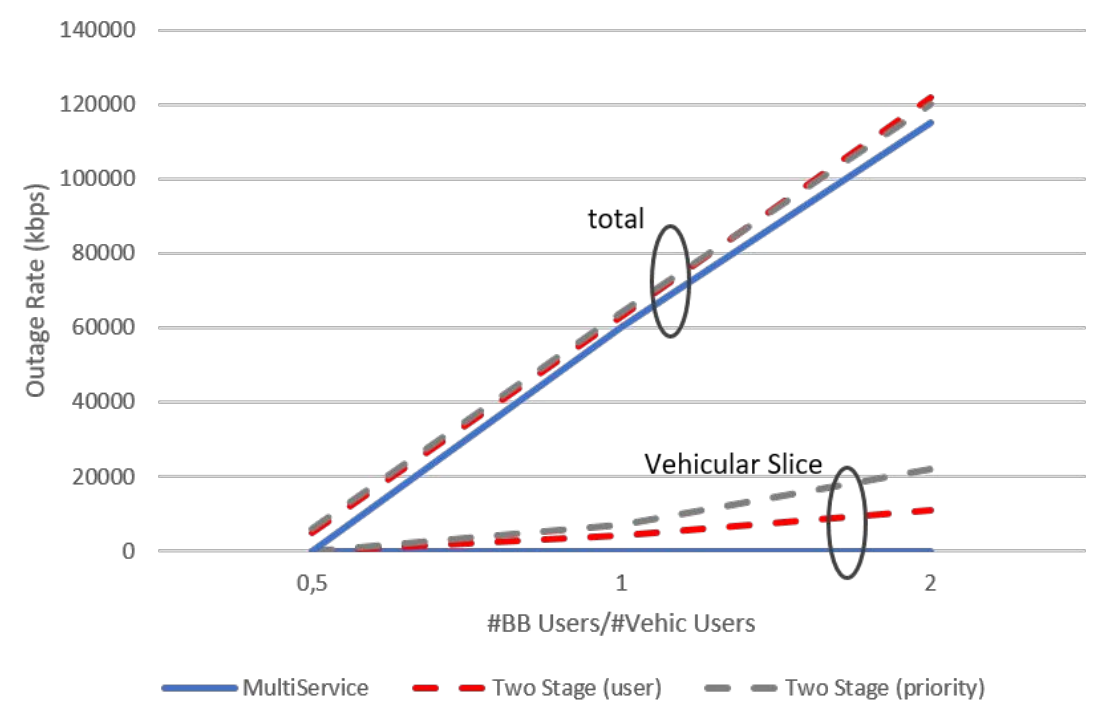

Figure 3. Outage rate of a two-slice system.

Multi RATs. The vehicles will be a powerful mobile gateway that incorporates multiple radio access technologies (Multi-RAT). Indeed, multi-RATs may be available for both V2V and vehicle-to-infrastructure (V2I) communications (e.g., 802.11p, LTE, C-V2X, 5G), which may need to be managed to exploit the benefits of each of them and to limit their drawbacks. In this context, one of the enhanced study/experimentations that is currently ongoing is the integration of visible light communication (VLC) in the proposed platform for V2I and V2V communications. Visible light represents a new communication opportunity for vehicular networking applications [28]. Compared to RF-based communications, VLC offers robustness against jamming attacks, a smaller interference domain, and a huge license-free spectrum. Energy efficiency is also addressed since two "services" (traffic guidance and communication) are implemented with the same energy. Figure 4 shows the preliminary elements coming from a study conducted at the University of Florence for deploying the experimentation activities in the city of Prato. A real traffic-light, transmitting a message via VLC, was positioned in a real road, and a car with a VLC receiver on the dashboard moved toward the light. A bit-rate of $200 \mathrm{kbps}$ was received up to $40 \mathrm{~m}$.

A custom LED driver was installed in a traffic light. The LED driver piloted the modulation of the light, thus inserting digital information. In particular, the LED driver moved the intensity of the LED to $+\Delta$ (bit 1 ) and $-\Delta$ (bit 0 ) around a mean value (the legal intensity of the traffic light). A traffic light lens 
is a typical legal traffic light. The traffic light was placed in a regular road at a typical distance from the lanes. A photodiode was used as the receiver, and a lens collected the light. The photodiode was wired to a high precision oscilloscope for the display and record of the VLC signal. The receiver was moved on a virtual grid from 2 to $40 \mathrm{~m}$ along the lanes road. In each point of the grid, the received VLC signal was recorded for $40 \mathrm{~s}$. The measurements led to a propagation model and a detailed performance evaluation.

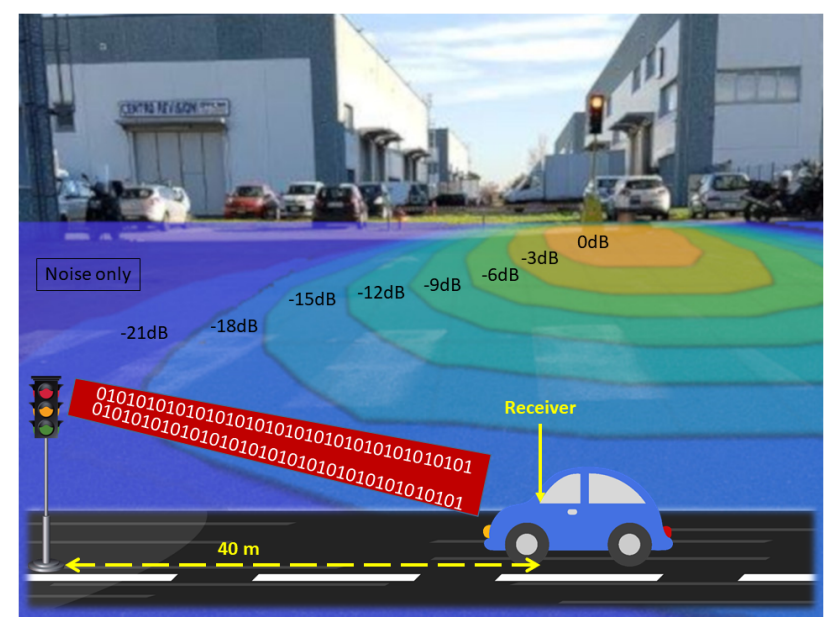

Figure 4. Experimentation of I2V link using visible light communication (VLC).

MEC. A multi-access edge computing paradigm allows cloud-computing capabilities to be deployed closer to the user within the RAN, thus reducing network congestion and improving application performance. MEC allows one to efficiently process data generated from different sources (vehicles and infrastructure) and to deliver locally relevant content for smart driving services. This environment was characterized by ultra-low latency, high bandwidth, and real-time access to the RAN information that can be leveraged by the applications.

Access point densification. In order to provide sufficient coverage and/or capacity, small cells have to be added to the network infrastructure, and some vehicles can complement the public network by becoming self-deployed moving cells (for example, in case of emergency or network unavailability). However, the coexistence of multiple cell-layers requires careful investigation on resource usage and generated interference, and hence requires coordination/cooperation among cells. An effective way to manage interference limiting complexity and signaling overhead is enhanced inter-cell interference coordination (eICIC) $[27,29]$. An extension of the eICIC concept is under evaluation in enhanced studies. In particular, we considered a moving small cell, deployed where and when needed to provide dedicated access to vehicular users, which operate on the same channel and on the same area of the macrocell belonging to the conventional network. The two cells access the resources on a time-division basis, in some time slots in exclusive mode (interference free slots, $N_{M}$ and $N_{S}$ ) and in others in jointly mode (slots affected by mutual interference $N_{J}$ ), see Figure 5 . The total capacity of the system is maximized if the joint allocation of users in the subframes and subframes distribution are suitably performed. For example, in comparison with a fixed partitioning of the slots among macrocells and moving small cells, we have a reduction of almost $50 \%$ of the outage rate in an overloaded situation. 


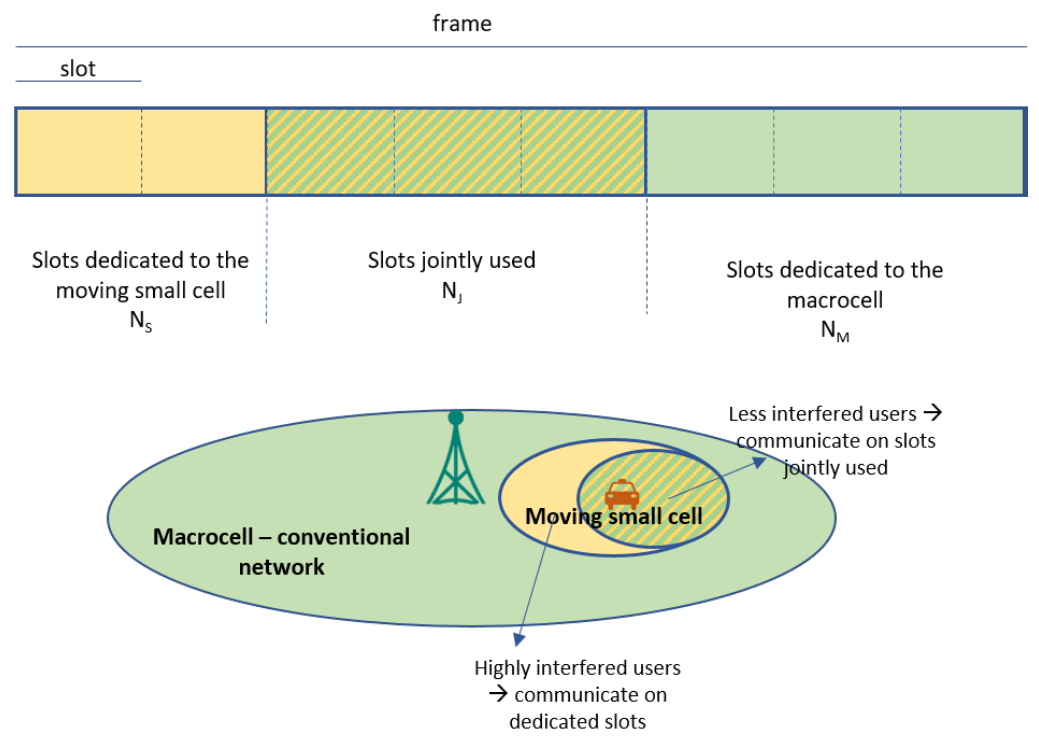

Figure 5. The eICIC concept extended to the coexistence of moving small cells and conventional macrocells.

\section{Conclusions}

In this the paper, we provide a detailed description of the deployment of a complete integrated 5G network in the city of Prato, Italy. Solutions, technologies, and scenarios and use cases are discussed. Key performance indicators, depending on the specific service, and preliminary results are also shown. The paper further describes how $5 \mathrm{G}$ is an enabling technology for a wide range of vertical business and services. The flexibility of the 5G network allows one to satisfy completely different performance requirements of real applications. This paper provides a complete view of how $5 \mathrm{G}$ technology can be implemented and which challenges need to be faced to enable new services in a real urban scenario.

Author Contributions: Conceptualization: D.M., L.M., and R.F.; data curation: D.M., L.M., M.R.S., and F.M.; formal analysis: D.M., L.M.; funding acquisition, M.R.S., F.M., A.F., and G.C.; investigation: D.M., L.M., and R.F.; methodology: D.M., L.M., and R.F.; supervision: D.M., L.M., R.F., M.R.S., F.M., A.F., G.C., J.Y., and L.F.; validation: M.R.S., F.M., A.F., G.C., J.Y., and L.F.; writing—original draft: D.M. and L.M.; writing—review \& editing: D.M., L.M., R.F., and M.R.S.

Funding: This research received no external funding.

Conflicts of Interest: The authors declare no conflict of interest.

\section{References}

1. Hammi, B.; Khatoun, R.; Zeadally, S.; Fayad, A.; Khoukhi, L. IoT technologies for smart cities. IEE Netw. 2018, 7, 1-13. [CrossRef]

2. Zaidi, A.A.; Baldemair, R.; Tullberg, H.; Bjorkegren, H.; Sundstrom, L.; Medbo, J.; Kilinc, C.; Silva, I.D. Waveform and numerology to support 5G services and requirements. IEEE Commun. Mag. 2016, 54, 90-98. [CrossRef]

3. Shafi, M.; Molisch, A.F.; Smith, P.J.; Haustein, T.; Zhu, P.; Silva, P.D.; Tufvesson, F.; Benjebbour, A.; Wunder, G. 5G: A Tutorial Overview of Standards, Trials, Challenges, Deployment, and Practice. IEEE J. Sel. Areas Commun. 2017, 35, 1201-1221. [CrossRef]

4. El Ayoubi, S.E.; Fallgren, M.; Spapis, P.; Qi, Y.; Martín-Sacristán, D.; Carrasco, Ó.; Fresia, M.; Payaró, M.; Schubert, M.; Bedo, J.S. 5G PPP Use Cases and Performance Evaluation Models. Available online: http: / /www.5g-ppp.eu/ (accessed on 25 April 2016).

5. Pätzold, M. 5G developments are in full swing [mobile radio]. IEEE Veh. Technol. Mag. 2017 12, 4-12. [CrossRef] 
6. Malkowsky, S.; Vieira, J.; Liu, L.; Nieman, K.; Kundargi, N.; Wong, I.; Tufvesson, F.; Öwall, V.; Edfors, O. Real-time testbed for massive MIMO: Design, implementation, and real-life validation. IEEE Access 20175 , 9073-9088. [CrossRef]

7. Jiang, X.; Kaltenberger, F.; Knopp, R.; Maatallah, H. OpenAirInterface massive MIMO testbed: A 5G innovation platform. In Proceedings of the 21st International ITG Workshop Smart Antennas, Berlin, Germany, 15-17 March 2017.

8. Oproiu, E.; Iordache, M.; Costea, C.; Brezeanu, C.; Patachia, C. 5G Network Architecture, Functional Model and Business Role for 5G Smart City Use Case: Mobile Operator Perspective. In Proceedings of the 2018 International Conference on Communications (COMM), Bucharest, Romania, 14-16 June 2018; pp. 361-366.

9. Rusti, B.; Stefanescu, H.; Ghenta, J.; Patachia, C. Smart City as a 5G Ready Application. In Proceedings of the 2018 International Conference on Communications (COMM), Bucharest, Romania, 14-16 June 2018; pp. 207-212.

10. Leligou, H.C.; Zahariadis, T.; Sarakis, L.; Tsampasis, E.; Voulkidis, A.; Velivassaki, T.E. Smart Grid: A demanding use case for $5 \mathrm{G}$ technologies. In Proceedings of the IEEE International Conference on Pervasive Computing and Communications Workshops (PerCom Workshops), Athens, Greece, 19-23 March 2018; pp. 215-220.

11. Bartoli, G.; Fantacci, R.; Gei, F.; Marabissi, D.; Micciullo, L. A Novel Emergency Management Platform for Smart Public Safety. Int. J. Commun. Syst. 2015, 28, 928-943. [CrossRef]

12. Carla, L.; Fantacci, R.; Gei, F.; Marabissi, D.; Micciullo, L. LTE enhancements for Public Safety and Security communications to support Group Multimedia Communications. IEEE Netw. 2016, 30, 80-85. [CrossRef]

13. Fantacci, R.; Gei, F.; Marabissi, D.; Micciullo, L. Public Safety Networks evolution towards broadband: Sharing infrastructures and spectrum with commercial systems. IEEE Commun. Mag. 2016, 54, 24-30. [CrossRef]

14. Elayoubi, S.E.; Fallgren, M.; Spapis, P.; Zimmermann, G.; Martín-Sacristán, D.; Yang, C.; Jeux, S.; Agyapong, P.; Campoy, L.; Qi, Y.; et al. 5G service requirements and operational use cases: Analysis and METIS II vision. In Proceedings of the 2016 European Conference on Networks and Communications (EuCNC), Athens, Greece, 27-30 June 2016; pp. 158-162. [CrossRef]

15. Gangakhedkar, S.; Cao, H.; Ali, A.R.; Ganesan, K.; Gharba, M.; Eichinger, J. Use Cases, Requirements and Challenges of $5 \mathrm{G}$ Communication for Industrial Automation. In Proceedings of the IEEE International Conference on Communications Workshops (ICC Workshops), Kansas City, MO, USA, 20-24 May 2018; pp. 1-6. [CrossRef]

16. Masini, B.M.; Bazzi, A.; Zanella, A. Survey on the Roadmap to Mandate on Board Connectivity and Enable V2V-Based Vehicular Sensor Networks. Sensors 2018, 18, 2207. [CrossRef] [PubMed]

17. Kanstrén, T.; Mäkelä, J.; Uitto, M.; Apilo, O.; Pouttu, A.; Liinamaa, O.; Destino, G.; Kivinen, P.; Matilainen, A. Vertical Use Cases in the Finnish 5G Test Network. In Proceedings of the European Conference on Networks and Communications (EuCNC), Ljubljana, Slovenia, 18-21 June 2018; pp. 329-334. [CrossRef]

18. Pouttu, A.; Liinamaa, O.; Destino, G. Demo/poster abstract: 5G test network (5GTN)-Environment for demonstrating 5G and IoT convergence during 2018 Korean Olympics between Finland and Korea. In Proceedings of the INFOCOM 2018-IEEE Conference on Computer Communications Workshops (INFOCOM WKSHPS), Honolulu, HI, USA, 15-19 April 2018; pp. 1-2. [CrossRef]

19. Lauridsen, M.; Gimenez, L.C.; Rodriguez, I.; Sorensen, T.B.; Mogensen, P. From LTE to 5G for Connected Mobility. IEEE Commun. Mag. 2017, 55, 156-162. [CrossRef]

20. Rost, P.; Mannweiler, C.; Michalopoulos, D.S.; Sartori, C.; Sciancalepore, V.; Sastry, N.; Holland, O.; Tayade, S.; Han, B.; Bega, D.; et al. Network slicing to enable scalability and flexibility in 5G mobile networks. IEEE Commun. Mag. 2017, 55, 72-79. [CrossRef]

21. De la Oliva, A.; Hernandez, J.A.; Larrabeiti, D.; Azcorra, A. An overview of the cpri specification and its application to c-ran-based lte scenarios. IEEE Commun. Mag. 2016, 54, 152-159. [CrossRef]

22. Optical Internetworking Forum. IA OIF-FLEXE Flex Ethernet Implementation Agreement; Technical Report 01.0. Available online: http:/ / www.oiforum.com/wp-content/uploads/OIF-FLEXE-01.0.pdf (accessed on 22 December 2018).

23. 3GPP Global Initiative. 3rd Generation Partnership Project. TR 23.799 Technical Specification Group Services and System Aspects; Study on Architecture for Next Generation System; Technical Report V14.0.0. Available online: http:/ / www.3gpp.org/DynaReport/23799.htm (accessed on 22 December 2018). 
24. Ordonez-Lucena, J.; Ameigeiras, P.; Lopez, D.; Ramos-Munoz, J.J.; Lorca, J.; Folgueira, J. Network slicing for 5G with sdn/nfv: Concepts, architectures, and challenges. IEEE Commun. Mag. 2017, 55, 80-87. [CrossRef]

25. International Telecommunciation Union. Recommendation ITU-R M.2083-0 IMT Vision-Framework and Overall Objectives of the Future Development of IMT for 2020 and Beyond; Technical Report M Series; Recommendation ITU: Geneva, Switzerland, 2015.

26. International Telecommunciation Union. Minimum Requirements Related to Technical Performance for IMT-2020 Radio Interface(s); Document ITU-R M.[IMT-2020.TECH PERF REQ]. Available online: https: / / www.itu.int/pub/R-REP-M.2410-2017 (accessed on 22 December 2018).

27. Marabissi, D.; Fantacci, R. Heterogeneous public safety network architecture based on ran slicing. IEEE Access 2018, 5, 668-677. [CrossRef]

28. Masini, B.M.; Bazzi, A.; Zanella, A. Vehicular visible light networks for urban mobile crowd sensing. Sensors 2018, 18, 1177. [CrossRef] [PubMed]

29. Marabissi, D.; Bartoli, G.; Fantacci, R.; Pucci, M. An optimized comp transmission for a heterogeneous network using eicic approach. IEEE Trans. Veh. Technol. 2016 65, 8230-8239. [CrossRef]

(C) 2018 by the authors. Licensee MDPI, Basel, Switzerland. This article is an open access article distributed under the terms and conditions of the Creative Commons Attribution (CC BY) license (http://creativecommons.org/licenses/by/4.0/). 